Palavras chave:

Biomassa

Bioenergia

Resíduos da cafeicultura

Histórico:

Recebido 06/07/201 I

Aceito 20/05/2014

Keywords:

Biomass

Bioenergy

Coffee planting residues

Correspondência: depaulaprotasio@gmail.com

DOI:
Edson Rubens da Silva Leite', Thiago de Paula Protásio², Sebastião Carlos da Silva Rosado ${ }^{3}$, Paulo Fernando Trugilho3 ${ }^{3}$ Gustavo Henrique Denzin Tonoli ${ }^{3}$, Lina Bufalino ${ }^{4}$

\section{AVALIAÇÃO DA QUALIDADE DA MADEIRA DE Coffea arabica L. COMO FONTE DE BIOENERGIA}

RESUMO: Objetivou-se, neste trabalho, avaliar, por meio da análise de componentes principais, a qualidade da madeira de Coffea arabica L. em diferentes variedades e sistemas de cultivo como fonte de bioenergia. Foram utilizadas madeiras provenientes de três sistemas de cultivo (natural, convencional e orgânico) e de duas variedades distintas (Mundo Novo e Catuaí), totalizando seis tratamentos. Foram quantificados os teores dos componentes elementares ( $, \mathrm{H}, \mathrm{N}, \mathrm{S}$ e $\mathrm{O}$ ), de cinzas, lignina, extrativos totais e holocelulose, a densidade básica, o poder calorífico superior, o poder calorífico inferior, o poder calorífico superior e inferior volumétricos das madeiras analisadas. Foi realizada a análise estatística multivariada de componentes principais. Os escores das componentes principais de interesse foram determinados como forma de dividir as madeiras em grupos. O grupo I foi composto pela madeira do cafeeiro orgânico Mundo Novo e natural Mundo Novo; o grupo II pelo orgânico Catuaí, e o grupo III pelas madeiras dos cafeeiros convencional Mundo Novo, natural Catuaí e convencional Catuaí. Os resíduos madeireiros da espécie Coffea arabica L. apresentaram grande potencial para o uso energético, com destaque para as madeiras provenientes do sistema de cultivo convencional e da variedade Catuaí. As madeiras do grupo III se destacaram em decorrência dos elevados valores de poder calorífico volumétrico, densidade básica, teor de lignina e poder calorífico superior. Entretanto, as madeiras desse grupo apresentaram elevado teor de nitrogênio.

\section{EVALUATION OF Coffea arabica L. WOOD QUALITY AS A SOURCE OF BIOENERGY}

ABSTRACT: This work aimed to evaluate, through principal component analysis, the quality of Coffea arabica L. wood obtained from different growth systems and varieties as a source of bioenergy. Wood from three different growth systems (natural, conventional and organic) and two different varieties (Mundo Novo and Catuaí) were used, totalizing six treatments. The contents of elementary components ( $\mathrm{C}, \mathrm{H}, \mathrm{N}, \mathrm{S}$ and $\mathrm{O}$ ), ash, lignin, total extractives and holocellulose, basic density, the higher heating value, the lower heating value, as well as the lower and higher volumetric heating values of the analyzed woods were quantified. Principal components multivariate statistical analyses were conducted. The scores of the principal components of interest were determined as a way to divide the woods into groups. Group I was composed by the wood from the organic coffee "Mundo Novo" and natural "Mundo Novo"; Group II by the organic "Catuaí", and Group III by the wood from convencional "Mundo Novo", natural "Catuaí" and convencional "Catuaí". Wood residues from the species Coffea arabica L. showed great potential for energetic use, especially the woods from the conventional systems and the "Catuaí" Variety. Woods from Group III stood out, due to the high volumetric heating values, basic density, lignin content and higher heating value. However, the woods of this group showed high nitrogen content.

I Instituto Federal de Educação, Ciência e Tecnologia do Sul de Minas Gerais - Machado, Minas Gerais, Brasil

2 Universidade Federal de Goiás - Jataí, Goiás, Brasil

${ }^{3}$ Universidade Federal de Lavras - Lavras, Minas Gerais, Brasil

${ }^{4}$ Universidade do Estado do Amapá - Macapá, Amapá, Brasil 


\section{INTRODUÇÃO}

Atualmente, tem-se buscado fontes alternativas de energia renovável, uma vez que a matriz energética mundial é altamente dependente dos combustíveis fósseis. A grande vantagem da utilização da biomassa vegetal como fonte de energia consiste nas emissões neutras de dióxido de carbono. $\mathrm{O} \mathrm{CO}_{2}$ produzido a partir da queima da biomassa (oxidação) é absorvido pelas plantas, por meio do processo fotossintético, que crescem em um ciclo relativamente curto (LÓPEZRODRÍGUEZ et al., 2009; MOGHTADERI et al., 2006; VOIVONTAS et al., 200I).

Por isso, é importante que os plantios sejam repostos, visando à reincorporação do $\mathrm{CO}_{2}$ emitido na biomassa vegetal, durante o crescimento das plantas e que esses biocombustíveis sejam utilizados próximos dos locais de colheita, evitando, assim, emissões de $\mathrm{CO}_{2} \mathrm{com}$ o transporte (PROTÁSIO et al., 20I3a).

Diante disso, o aproveitamento da biomassa residual advinda da cafeicultura pode ser vantajoso do ponto de vista econômico e ambiental, pois esses resíduos apresentam baixo custo e significativa disponibilidade.

O Brasil se destaca no cenário internacional como grande produtor de café. Em 2013, o país deve colher entre 46,98 e 50,16 milhões de sacas de 60 quilos do produto beneficiado, em uma área estimada de 2.375,79 mil hectares (COMPANHIA NACIONAL DE ABASTECIMENTO - CONAB, 20I3), sendo adotadas várias técnicas de manejo e sistemas de cultivos específicos para cada localidade ou tipo de solo que podem influenciar na qualidade e no rendimento energético da madeira do cafeeiro. $O$ sistema de cultivo natural caracteriza-se pela pouca interferência humana na lavoura, ou seja, não são realizadas adubações químicas, aplicações de herbicidas ou inseticidas e podas regulares. Já, o sistema de cultivo convencional é amplamente utilizado no Brasil e segue os preceitos clássicos da cafeicultura. O sistema de cultivo orgânico visa à diminuição dos resíduos de agrotóxicos e uma diversificação da cafeicultura, por meio de consórcios em sistemas agroflorestais, pois se fundamenta em princípios agroecológicos e de conservação dos recursos naturais (THEODORO et al., 2003).

A grande produção brasileira de café resulta em grande quantidade de resíduos lignocelulósicos provenientes do processamento dos grãos, bem como das renovações das lavouras cafeeiras, que apresentam potencial para a produção de bioenergia. Contudo, é fundamental a avaliação da composição química, física e energética da biomassa, pois os cálculos de rendimento dos sistemas de combustão e eficiência dos projetos de geração de energia dependem de características como, por exemplo, poder calorífico, densidade, teores de lignina, extrativos totais, carbono, oxigênio, hidrogênio, materiais voláteis e cinzas.

Nesse contexto, o poder calorífico é uma das principais características avaliadas na biomassa para o uso energético, pois expressa a quantidade de calor total (energia térmica) que é liberado durante a combustão completa de uma unidade de massa ou de volume do material combustível. Contudo, o poder calorífico é amplamente influenciado pela composição química e tipo de biomassa, umidade, carbono fixo e cinzas (BRAND, 20I0; DEMIRBAS, 2004; EROL et al., 20I0; PROTÁSIO et al., 20II, 20I2b; REIS et al., 20I2b; SHENG; AZEVEDO, 2005).

Diante da ausência na literatura de estudos relacionados à avaliação do potencial energético da madeira de Coffea arabica L., objetivou-se, neste trabalho, avaliar, por meio da análise de componentes principais, a qualidade da madeira de Coffea arabica L. de diferentes variedades e sistemas de cultivo como fonte de bioenergia.

\section{MATERIAL E MÉTODOS}

\section{Caracterização química, física e energética da madeira}

Foram utilizadas madeiras de Coffea arabica L. provenientes de três sistemas de cultivo (natural, convencional e orgânico) e de duas variedades distintas (Mundo Novo e Catuaí), totalizando seis tratamentos, ou seja, os cafeeiros natural Mundo Novo (NtMN), natural Catuaí ( $\mathrm{NtC})$, convencional Mundo Novo (ConvMN), convencional Catuaí (ConvC), orgânico Mundo Novo (OrgMN) e orgânico Catuaí (OrgC).

Foram amostradas aleatoriamente quatro repetições, com idade de aproximadamente 10 anos, resultando em 24 arbustos abatidos. De cada arbusto, foram retirados dois discos de aproximadamente $5 \mathrm{~cm}$ de espessura, sendo um obtido na base para determinação da densidade básica e outro a $30 \mathrm{~cm}$ de altura do solo, destinado às análises químicas e energéticas.

As madeiras foram coletadas no câmpus do Instituto Federal de Educação, Ciência e Tecnologia do Sul de Minas Gerais no município de Machado (MG), latitude $21^{\circ} 40^{\prime} \mathrm{S}$, longitude $45^{\circ} 59^{\prime} \mathrm{W}$ e altitude de $873 \mathrm{~m}$. 
$\mathrm{Na}$ determinação da densidade básica da madeira, foi utilizado o método de imersão, segundo a norma NBR II94I (ASSOCIAÇÃO BRASILEIRA DE NORMAS TÉCNICAS - ABNT, 2003).

$O$ teor de cinzas foi determinado segundo as diretrizes previstas na norma MII/77 (ASSOCIAÇÃO BRASILEIRA TÉCNICA DE CELULOSE E PAPEL ABTCP, 1974b) com o uso de um forno tipo mufla. Os extrativos totais e a lignina insolúvel da biomassa avaliada foram quantificados, seguindo as determinações das normas M3/69 (ABTCP, 1974a) e M70/7I(ABTCP, 1974c), respectivamente. $O$ teor de holocelulose foi obtido pela subtração dos teores de cinzas, extrativos totais e lignina da massa seca total da biomassa utilizada.

A análise elementar foi realizada em um analisador universal da marca Elementar (modelo Vario Micro Cube), para a quantificação dos teores de carbono, hidrogênio, nitrogênio e enxofre em relação à massa seca dos resíduos lenhosos da cafeicultura. As amostras foram moídas e peneiradas, sendo utilizadas para o ensaio $2 \mathrm{mg}$ da fração retida na peneira de 270 mesh, conforme utilizado por Neves et al. (20I I) e Protásio et al. (20II, 2012a, 20I3a). O teor de oxigênio foi obtido por diferença, segundo a equação I (BECH et al., 2009), sendo $\mathrm{O}=$ teor de oxigênio (\%); $\mathrm{C}=$ teor de carbono (\%); $\mathrm{H}=$ teor de hidrogênio (\%); $\mathrm{N}=$ teor de nitrogênio (\%); $\mathrm{S}=$ teor de enxofre (\%) e $\mathrm{Cz}=$ teor de cinzas (\%).

$\mathrm{O}=\mathrm{I} 00-\mathrm{C}-\mathrm{H}-\mathrm{N}-\mathrm{S}-\mathrm{Cz}$

Para a quantificação do poder calorífico superior (PCS), foi utilizado um calorímetro digital da marca IKA C-200, de acordo com os procedimentos descritos na NBR 8633 (ABNT, 1984). As amostras para a determinação do PCS foram classificadas em peneiras de 40/60 mesh, sendo utilizadas, no ensaio, as frações das amostras retidas na peneira de 60 mesh, as quais foram secas em estufa a $103 \pm 2{ }^{\circ} \mathrm{C}$ até massa constante. O poder calorífico inferior a $0 \%$ de umidade foi estimado utilizando a equação 2 , sendo $\mathrm{PCl}=$ poder calorífico inferior $\left(\mathrm{kcal} \cdot \mathrm{kg}^{-1}\right)$; PCS = poder calorífico superior $(\mathrm{kcal}$. $\mathrm{kg}^{-1}$ ) e $\mathrm{H}=$ teor de hidrogênio (\%).

$\mathrm{PCI}=\mathrm{PCS}-\left(600 \cdot \frac{9 \mathrm{H}}{100}\right)$

O poder calorífico superior volumétrico (PCSv) e o poder calorífico inferior volumétrico ( $\mathrm{PClv}$ ) foram calculados multiplicando-se a densidade básica da madeira pelo poder calorífico superior (PCS) e inferior $(\mathrm{PCl})$, respectivamente (BRAND, 20I0).

\section{Análise de componentes principais}

Foram consideradas apenas as médias de cada variável e a análise de componentes principais foi realizada, considerando-se a matriz de correlação dos dados. Esse procedimento equivale a padronizar as variáveis e permite maior acurácia na análise (MINGOTI, 2005). Foram determinados os escores das componentes principais de interesse como forma de classificação e formação de grupos da madeira avaliada.

Todas as análises estatísticas foram efetuadas, utilizando-se o software $R$ versão 2. II.0, pacote stats ( $R$ DEVELOPMENT CORE TEAM, 20I0).

\section{RESULTADOS E DISCUSSÃO}

Observou-se que as três primeiras componentes principais explicaram $89,49 \%$ da variância total. Portanto, as informações mais relevantes dos dados amostrais originais estão contidas nessas três componentes.

$\mathrm{Na}$ Tabela I, encontram-se os autovetores e as correlações entre as variáveis originais e as três primeiras componentes principais (CPI, CP2 e CP3) consideradas.

TABELA I Autovetores (ê) e as correlações ( $r$ ) entre as variáveis originais e as 3 primeiras componentes principais.

TABLE I Eigenvectors (ê) and correlations ( $r$ ) between original variables and the first 3 principal components.

\begin{tabular}{lcccccc}
\hline \multirow{2}{*}{ Variáveis } & \multicolumn{2}{c}{ CPI } & \multicolumn{2}{c}{ CP2 } & \multicolumn{2}{c}{ CP3 } \\
\cline { 2 - 7 } & $\hat{e}_{1}$ & $r$ & $\hat{e}_{2}$ & $r$ & $\hat{e}_{3}$ & $r$ \\
\hline Extrativos totais & $-0,349$ & $-0,84$ & $-0,208$ & $-0,45$ & 0,161 & 0,24 \\
Lignina insolúvel & 0,316 & 0,76 & $-0,189$ & $-0,40$ & 0,265 & 0,39 \\
Nitrogênio & 0,255 & 0,61 & 0,070 & 0,15 & 0,467 & 0,69 \\
Hidrogênio & $-0,141$ & $-0,34$ & 0,290 & 0,62 & 0,224 & 0,33 \\
Carbono & $-0,252$ & $-0,60$ & 0,329 & 0,70 & 0,180 & 0,27 \\
Enxofre & 0,113 & 0,27 & $-0,110$ & $-0,23$ & 0,587 & 0,87 \\
Oxigênio & 0,100 & 0,24 & $-0,429$ & $-0,92$ & $-0,146$ & $-0,22$ \\
Cinzas & 0,224 & 0,53 & 0,328 & 0,70 & $-0,286$ & $-0,42$ \\
PCI & 0,013 & 0,03 & $-0,404$ & $-0,86$ & $-0,173$ & $-0,26$ \\
Densidade básica & 0,413 & 0,99 & 0,025 & 0,05 & 0,019 & 0,03 \\
PClv & 0,412 & 0,98 & $-0,003$ & $-0,01$ & 0,006 & 0,01 \\
Holocelulose & 0,216 & 0,52 & 0,331 & 0,71 & $-0,319$ & $-0,47$ \\
PCSv & 0,411 & 0,98 & $-0,005$ & $-0,01$ & 0,006 & 0,01 \\
PCS & $-0,004$ & $-0,01$ & $-0,383$ & $-0,82$ & $-0,151$ & $-0,22$ \\
\hline
\end{tabular}

PCl: poder calorífico inferior; PClv: poder calorífico inferior volumétrico; PCSv: poder calorífico superior volumétrico; PCS: poder calorífico superior. 
A componente principal I encontra-se altamente correlacionada com a densidade básica da madeira, o teor de lignina insolúvel, o PCSv e o PClv e pode ser interpretada como um índice de rendimento energético. Quanto maiores os valores dessas variáveis maior será o valor numérico (escore) da componente principal I (CPI) e mais propício será o material para a produção de bioenergia.

Analisando-se a segunda componente principal (CP2), pode-se perceber que os maiores coeficientes, em módulo, referem-se aos teores de carbono, cinzas, oxigênio, poder calorífico inferior ( $\mathrm{PCl})$, poder calorífico superior (PCS) e holocelulose. Contudo, o maior coeficiente refere-se ao poder calorífico inferior, característica de grande importância na avaliação da biomassa para a produção energética. Quanto maiores os teores de carbono, cinzas e holocelulose maior será o valor numérico dessa componente principal, sendo o contrário verificado para o teor de oxigênio e para o $\mathrm{PCl}$ e PCS.

A terceira componente principal (CP3) pode ser interpretada como um índice de poluição ambiental e não aproveitamento energético da biomassa, pois os maiores coeficientes dessa componente são relativos aos teores de nitrogênio e enxofre.

$\mathrm{Na}$ Figura I, encontram-se o diagrama de ordenação das variáveis originais e os escores das componentes principais I e 2 . Um ponto qualquer plotado no diagrama (representando a madeira proveniente de um sistema de cultivo e de uma variedade de Coffea arabica L.) pode ser relacionado com cada seta (representando as variáveis originais observadas) (ALVARENGA; DAVIDE, 1999).

Observa-se que se pode formar três grupos distintos, considerando-se as componentes principais I e 2 que explicam $74 \%$ da variabilidade dos dados. Além disso, as variáveis mais importantes para a classificação e seleção da biomassa para a geração de bioenergia apresentaram maior contribuição nessas duas componentes.

O grupo I é formado pela madeira do cafeeiro orgânico Mundo Novo e natural Mundo Novo; o grupo II pelo orgânico Catuaí, e o grupo III pelas madeiras provenientes dos cafeeiros convencional Mundo Novo, natural Catuaí e convencional Catuaí.

Para a primeira componente principal (CPI), pode-se perceber que as madeiras do cafeeiro convencional Mundo Novo (ConvMN), natural Catuaí $(\mathrm{NtC})$ e convencional Catuaí (ConvC), pertencentes ao grupo III, apresentaram os maiores escores em

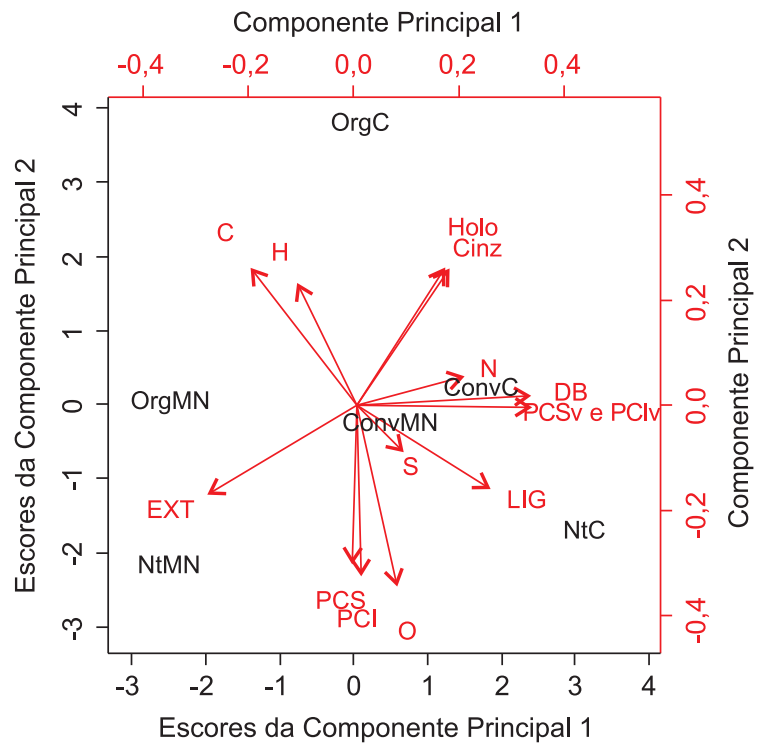

C: carbono; H: hidrogênio; N: nitrogênio; S: enxofre; Holo: holocelulose; Cinz: cinzas; DB: densidade básica; PCS: poder calorífico superior; $\mathrm{PCl}$ : poder calorífico inferior; $\mathrm{PCSv}$ : poder calorífico superior volumétrico; PClv: poder calorífico inferior volumétrico; LIG: lignina; EXT: extrativos totais.

FIGURA I Diagrama de ordenação das variáveis originais e escores das duas primeiras componentes principais.

FIGURE I Diagram of original variables ordination and scores of the first two principal components.

decorrência dos elevados valores de densidade básica, lignina, PCSv e PClv. O mesmo não foi observado para as madeiras do grupo I, em razão dos menores valores dessas características. Esse resultado é indicativo de que as madeiras provenientes do grupo III são mais propícias à produção de bioenergia.

Observa-se, ainda, que as madeiras do grupo I, natural Mundo Novo e orgânico Mundo Novo, apresentaram altos teores de extrativos totais. Os extrativos exercem importante papel na utilização da madeira, pois influenciam as suas propriedades físicas e energéticas (PROTÁSIO et al., 2012b; TELMO; LOUSADA, 20Il; VARGAS-MORENO et al., 2012) e dependendo da sua estabilidade térmica, essas substâncias podem contribuir para $\bigcirc$ aumento do rendimento em carvão vegetal (PROTÁSIO et al., 20I 2a; SANTOS et al., 20II).

Nota-se que a madeira da espécie Coffea arabica L. advinda do sistema orgânico variedade Catuaí (grupo II) apresentou os menores valores de PCS e $\mathrm{PCl}$ e o menor teor de oxigênio, mas não os menores teores de carbono, holocelulose e cinzas, quando comparadas às demais madeiras, resultando em elevado escore para a componente principal 2 . 
$\mathrm{Na}$ Tabela 2, encontram-se os valores médios das características observadas para os grupos formados pelas componentes principais I e 2, bem como os coeficientes de variação encontrados.

TABELA 2 Valores médios das características observadas para os grupos formados pela análise de componentes principais, bem como os coeficientes de variação encontrados (entre parênteses).

TABLE 2 Mean values of the characteristics observed for groups made by grouping analysis, as well as the variation coefficients found (between parentheses).

\begin{tabular}{|c|c|c|c|}
\hline Características & Grupo I & Grupo II & Grupo III \\
\hline Extrativos totais (\%) & $17,24_{(3,30)}$ & $6,7 I_{(5,44)}$ & $9,13_{(23,08)}$ \\
\hline Lignina (\%) & $30,95_{(17,01)}$ & $30,04_{(7,44)}$ & $34,04_{(5,50)}$ \\
\hline Holocelulose (\%) & $50,63_{(10,70)}$ & $61,48_{(3,45)}$ & $55,42_{(2,31)}$ \\
\hline Nitrogênio (\%) & $0,39_{(23,34)}$ & $0,42_{(6,44)}$ & $0,52_{(16,03)}$ \\
\hline Hidrogênio (\%) & $6,60_{(1,36)}$ & $6,64_{(1,27)}$ & $6,60_{(1,18)}$ \\
\hline Carbono (\%) & $49,68_{(1,38)}$ & $50,07_{(1,19)}$ & $49,\left.3\right|_{(0,84)}$ \\
\hline Enxc & $0,04_{(30,46)}$ & $0,03_{(20,33)}$ & $0,04_{(15,75)}$ \\
\hline Oxigênio (\%) & $42, I I_{(1,83)}$ & $41,07_{(1,52)}$ & $42, I I_{(1,17)}$ \\
\hline Cinzas (\%) & $\mathrm{I}, 18_{(9,52)}$ & $\mathrm{I}, 77_{(14,56)}$ & I,42 \\
\hline PCS $\left(\mathrm{kcal} \cdot \mathrm{kg}^{-1}\right)$ & $4673_{(0,53)}$ & $4627_{(0,63)}$ & $4675_{(0,37)}$ \\
\hline $\mathrm{PCl}\left(\mathrm{kcal} \cdot \mathrm{kg}^{-1}\right)$ & $4316_{(0,56)}$ & $4268_{(0,75)}$ & $4319_{(0,37)}$ \\
\hline Densidade básica $\left(\mathrm{kg} \cdot \mathrm{m}^{-3}\right)$ & $610_{(2,45)}$ & $660_{(1,70)}$ & $697_{(2,19)}$ \\
\hline PCSv $\left(\mathrm{Gcal} \cdot \mathrm{m}^{-3}\right)$ & $2,85_{(2,85)}$ & $3,05_{(2,20)}$ & $3,26_{(1,88)}$ \\
\hline PClv $\left(\right.$ Gcal $\left.\cdot \mathrm{m}^{-3}\right)$ & $2,63_{(2,86)}$ & $2,82_{(2,29)}$ & $3,01_{(1,94)}$ \\
\hline
\end{tabular}

PCS: poder calorífico superior; $\mathrm{PCl}$ : poder calorífico inferior; PCSv: poder calorífico superior volumétrico; PClv: poder calorífico inferior volumétrico.

Observa-se que, em média, os três grupos formados apresentaram teores de carbono, hidrogênio, oxigênio e enxofre, bem como de poder calorífico superior e inferior similares.

Para a produção de bioenergia é desejável que a madeira apresente altos teores de carbono e hidrogênio e baixos teores de oxigênio e cinzas, em decorrência das relações existentes entre esses componentes elementares e o poder calorífico (DEMIRBAS; DEMIRBAS, 2004; HUANG et al., 2009; PAULA et al., 20II; PROTÁSIO et al., 20I I).

Observou-se que as madeiras pertencentes ao grupo I apresentaram altos teores de extrativos totais e baixo teor de holocelulose, o que pode ser benéfico para a produção energética (DEMIRBAS, 200I, 2004; PROTÁSIO et al., 2012a). A holocelulose é menos estável termicamente e não contribui para o aumento do rendimento em carvão vegetal (PROTÁSIO et al., 20I2a). Contudo, esse grupo apresentou valores de densidade básica, PCSv e PClv inferiores aos demais grupos, o que resulta em um menor rendimento energético da biomassa.

O grupo II, formado pela madeira do cafeeiro orgânico Catuaí, ocupou posição intermediária quanto ao seu aproveitamento energético, pois apresentou os menores valores para $\circ \mathrm{PCS}$ e para $\circ \mathrm{PCl}$ e maiores valores de cinzas e holocelulose quando comparado às demais biomassas analisadas, mas não apresentou a menor densidade básica, resultando, assim, em maiores valores de PCSv e o PClv em relação ao grupo I.

O grupo III, formado pelas madeiras provenientes dos cafeeiros convencional Mundo Novo, natural Catuaí e convencional Catuaí, apresentou elevado potencial para a produção de bioenergia, em razão dos altos valores de densidade básica, PCSv, PClv e lignina em relação aos demais grupos. Dessa forma, a madeira proveniente desse grupo apresenta grande potencial para a produção de carvão vegetal, em razão da maior densidade básica e ao elevado teor de lignina (PROTÁSIO et al., 20 I2a; TRUGILHO et al., 1997).

O valor médio encontrado para a densidade básica das madeiras de Coffea arabica L. do grupo III foi superior ao encontrado para diferentes espécies e clones do gênero Eucalyptus (NEVES et al., 20I I; PROTÁSIO et al., 20I3b; REIS et al., 20I2a, 20I2b; RODRIGUES et al., 2008; TRUGILHO et al., 200I, 2003) e reforçam o grande potencial energético da biomassa analisada. Sabe-se que, quanto maior a densidade básica maior será a disponibilidade de energia por unidade de volume de madeira e maior será a densidade relativa aparente do carvão vegetal.

No entanto, o grupo III apresentou teor de nitrogênio superior aos demais e à média geral determinada por Brand (2010) para madeiras de folhosas $(0,30 \%)$. Para a produção de bioenergia, é desejável que a biomassa apresente baixas quantidades de nitrogênio, pois esse constituinte pode resultar em poluição ambiental, com a formação de óxidos de nitrogênio tóxicos, formação de chuva ácida e corrosão, após a combustão (BILGEN; KAYGUSUZ, 2008; KUMAR et al., 2010 ), além de não apresentar correlação positiva com o poder calorífico (HUANG et al., 2009).

Já, os baixos teores de enxofre encontrados para a madeira do cafeeiro dos diferentes grupos não comprometem a utilização energética dessa biomassa.

Os teores de cinzas observados para a madeira do cafeeiro dos três grupos formados foram superiores ao comumente encontrado $(0,04 \%$ a $1,00 \%)$ para a madeira das espécies/clones pertencentes ao gênero Eucalyptus estudadas por: Caixeta et al. (2003), Mori et al. (2003), Neves et al. (20I I, 2013), Protásio et al. (20II, 20I2b, 20l3b) e Trugilho et al. (200I, 2003). 
Isso ocorreu, possivelmente, aos tratos culturais empregados, como a adubação diferenciada, a própria fisiologia e metabolismo das espécies, bem como às diferenças nutricionais dos solos em que essas biomassas foram estabelecidas.

O elevado teor de cinzas é desvantajoso, porque diminui a transferência de calor no combustível e aumenta a corrosão dos equipamentos utilizados no processo, uma vez que os minerais não participam da combustão da biomassa (TAN; LAGERKVIST, 20II). Além disso, o alto teor de cinzas no carvão vegetal utilizado na siderurgia, proveniente de uma madeira com maior teor de minerais, pode provocar o acúmulo de impurezas no centro das peças do metal solidificado, promovendo variações nas propriedades do ferro-gusa, aço ou ferro-liga (ASSIS et al., 20I2; NEVES et al., 20I I).

Os teores de lignina e extrativos totais encontrados neste trabalho foram superiores ao relatado por Trugilho et al. (1996) para a espécie Eucalyptus saligna em diferentes idades. Os valores médios de lignina encontrados por Caixeta et al. (2003) para genótipos do gênero Eucalyptus (28,96\%) e por Gomide et al. (2005) para clones de Eucalyptus $(29,30 \%)$ também foram inferiores ao determinado para a madeira do cafeeiro (32,34\%).

Protásio et al. (20|3b) e Trugilho et al. (2003) encontraram valores médios de lignina total de 31,77\% e $31,41 \%$ para diferentes clones de Eucalyptus, respectivamente, corroborando ao observado para as madeiras de Coffea arabica L. analisadas.

Dentre os componentes químicos, a lignina é a que apresenta a maior estabilidade térmica, pelas ligações carbono-carbono entre as unidades monoméricas de fenil-propano e, consequentemente, a estabilidade de sua matriz aromática (SHARMA et al., 2004; YANG et al., 2006). Logo, quanto maior o teor de lignina mais indicada é a madeira para a produção de carvão vegetal (PROTÁSIO et al., 20I2a).

O poder calorífico superior determinado por Protásio et al. (20l2b) para os seguintes combustíveis de biomassa: maravalhas de eucalipto, bagaço de canade-açúcar, polpa celulósica de bambu, casca de café, resíduos da colheita do milho e casca de arroz foi inferior ao relatado nesse trabalho para a madeira do cafeeiro proveniente dos três grupos formados. Isso ocorreu, possivelmente, em razão dos maiores valores de lignina e extrativos totais, conforme relatado por: Demirbas (200 I , 2004), Protásio et al. (20I2a) e Telmo e Lousada (20II), observados para a madeira do cafeeiro, em relação ao observado por Protásio et al. (20l2b) para as biomassas citadas.
O poder calorífico superior volumétrico, observado para as madeiras dos três grupos formados, foi superior ao relatado por Lima et al. (20II) para a espécie Eucalyptus benthamii aos 6 anos de idade de 2,22 Gcal $\cdot \mathrm{m}^{-3}$ e por Protásio et al. (20l3b) para clones de Eucalyptus grandis e Eucalyptus urophylla aos 3,5 anos de idade de 2,38 $\mathrm{Gcal} \cdot \mathrm{m}^{-3}$. Vale et al. (200I) encontraram poder calorífico superior volumétrico médio de 3,17 Gcal $\cdot \mathrm{m}^{-3}$ para o carvão vegetal de dez espécies do cerrado utilizadas como fonte de bioenergia, assemelhando-se ao encontrado para o grupo III. Esses resultados são indicativos do potencial energético dos resíduos madeireiros da cafeicultura.

$\mathrm{Na}$ Figura 2, encontram-se o diagrama de ordenação das variáveis originais e os escores das componentes principais 2 e 3 .

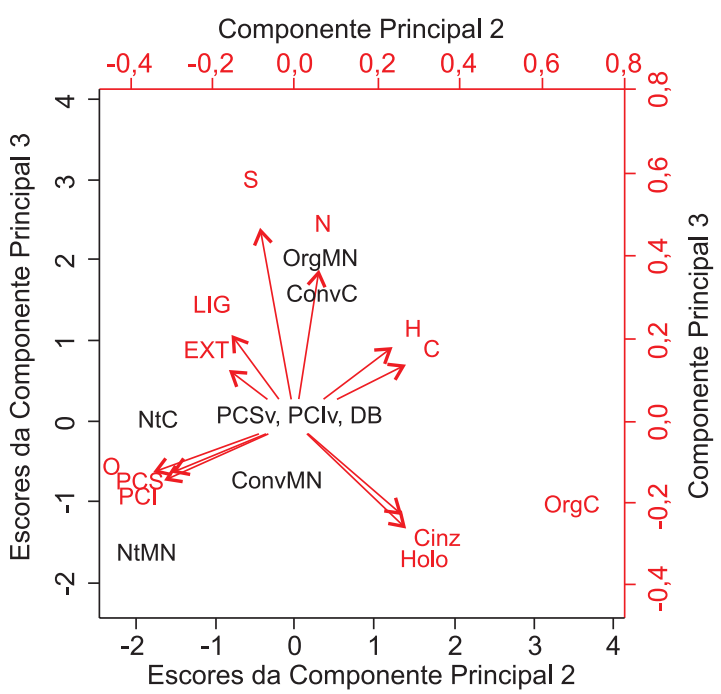

C: carbono; H: hidrogênio; $\mathrm{N}$ : nitrogênio; S: enxofre; Holo: holocelulose; Cinz: cinzas; DB: densidade básica; PCS: poder calorífico superior; PCI: poder calorífico inferior; PCSv: poder calorífico superior volumétrico; PClv: poder calorífico inferior volumétrico; LIG: lignina; EXT: extrativos totais.

FIGURA 2 Diagrama de ordenação das variáveis originais e escores das componentes principais 2 e 3 .

FIGURE 2 Diagram of original variables ordination and scores of principal components 2 and 3 .

Observa-se que as madeiras dos cafeeiros orgânicos Mundo Novo (OrgMN) e convencional Catuaí (ConvC) apresentaram elevados valores numéricos para a componente principal 3 , em razão dos altos teores de nitrogênio que podem dificultar a utilização energética da biomassa e levar a riscos de poluição ambiental. As demais madeiras analisadas apresentaram baixos escores para a componente principal 3, evidenciando baixos teores de nitrogênio e enxofre. 
Encontrou-se alta densidade básica, alto poder calorífico superior e inferior e um elevado poder calorífico volumétrico para as madeiras de Coffea arabica L. em relação às espécies/clones de Eucalyptus spp comumente utilizados na geração de bioenergia, com destaque para o sistema convencional e a variedade Catuaí. Isso pode resultar em bons rendimentos energéticos em sistemas de geração de energia como biotermelétricas ou gaseificadores.

Além disso, os resíduos madeireiros da cafeicultura, utilizados no presente trabalho apresentaram altos teores de hidrogênio, lignina e extrativos totais. Os resultados encontrados demonstram a potencialidade de utilização energética da madeira do cafeeiro e que pode resultar em um melhor aproveitamento ambiental e econômico dessa biomassa residual.

\section{CONCLUSÕES}

Os resíduos madeireiros da espécie Coffea arabica L. apresentaram grande potencial para $\circ$ uso energético, em razão dos altos teores de extrativos totais, lignina, carbono e elevada densidade básica e poder calorífico volumétrico, com destaque para as madeiras provenientes do sistema de cultivo convencional e da variedade Catuaí.

A técnica multivariada de componentes principais foi eficiente na análise bioenergética das madeiras de Coffea arabica L., sendo obtidos grupos homogêneos das amostras avaliadas. As madeiras do grupo III (convencional Mundo Novo, natural Catuaí e convencional Catuaí) destacaram-se, como fonte direta de bioenergia ou para a produção de carvão vegetal, em razão dos elevados valores de poder calorífico volumétrico, densidade básica, poder calorífico superior e teor de lignina.

\section{REFERÊNCIAS}

ALVARENGA, M. I. N.; DAVIDE, A. C. Características físicas e químicas de um Latossolo Vermelho-Escuro e a sustentabilidade de agroecossistemas. Revista Brasileira de Ciência do Solo, Viçosa, v. 23, p. 933-942, 1999.

ASSIS, M. R.; PROTÁSIO, T. P.; ASSIS, C. O.; TRUGILHO, P. F.; SANTANA, W. M. S. Qualidade e rendimentos do carvão vegetal de um clone híbrido de Eucalyptus grandis $x$ Eucalyptus urophylla. Pesquisa Florestal Brasileira, Colombo, v. 32, n. 7I, p. 29I-302, 2012.

ASSOCIAÇÃO BRASILEIRA DE NORMAS TÉCNICAS. NBR 8633: carvão vegetal: determinação do poder calorífico. Rio de Janeiro, 1984.
ASSOCIAÇÃO BRASILEIRA DE NORMAS TÉCNICAS. NBR I 194 | : madeira: determinação da densidade básica. Rio de Janeiro, 2003. 6 p.

ASSOCIAÇÃO BRASILEIRA TÉCNICA DE CELULOSE E PAPEL. M3/69: métodos de ensaio. São Paulo, 1974a.

ASSOCIAÇÃO BRASILEIRA TÉCNICA DE CELULOSE E PAPEL. M-I I/77: teor de cinzas. São Paulo, 1974b. 8 p.

ASSOCIAÇÃO BRASILEIRA TÉCNICA DE CELULOSE E PAPEL. M70/7 I: métodos de ensaio. São Paulo, 1974c.

BECH, N.; JENSEN, P. A.; DAM-JOHANSEN, K. Determining the elemental composition of fuels by bomb calorimetry and the inverse correlation of HHV with elemental composition. Biomass and Bioenergy, Oxford, v. 33, n. 3, p. 534-537, 2009.

BILGEN, S.; KAYGUSUZ, K. The calculation of the chemical exergies of coal-based fuels by using the higher heating values. Applied Energy, London, v. 85, n. 8, p. 776-785, 2008.

BRAND, M. A. Energia de biomassa florestal. Rio de Janeiro: Interciência, 20I0. I3I p.

CAIXETA, R. P.; TRUGILHO, P. F.; ROSADO, S. C. S.; LIMA, J. T. Propriedades e classificação da madeira aplicadas à seleção de genótipos de Eucalyptus. Revista Árvore, Viçosa, v. 27, n. I, p. 43-5I, 2003.

COMPANHIA NACIONAL DE ABASTECIMENTO. Avaliação da safra agrícola cafeeira: $I^{\mathrm{a}}$ estimativa, janeiro/20I3. Brasília, 2013. 19 p.

DEMIRBAS, A. Relationships between heating value and lignin, moisture, ash and extractive contents of biomass fuels. Journal Energy, Exploration \& Exploitation, Brentwood, v. 22, n. 2, p. I35-|43, 2004.

DEMIRBAS, A. Relationships between lignin contents and heating values of biomass. Energy Conversion and Management, Amsterdam, v. 42, n. 2, p. I83-I88, 2001.

DEMIRBAS, A.; DEMIRBAS, H. A. Estimating the calorific values of lignocellulosic fuels. Journal Energy, Exploration \& Exploitation, Brentwood, v. 20, n. I, p. |05-III, 2004.

EROL, M.; HAYKIRI-ACMA, H.; KUÇUKBAYRAK, S. Calorific value estimation of biomass from their proximate analyses data. Renewable Energy, Oxford, v. 35, n. I, p. I70-I73, 2010.

GOMIDE, J. L.; COLODETTE, J. L.; OLIVEIRA, R. C.; SILVA, C. M. Caracterização tecnológica, para produção de celulose, da nova geração de clones de Eucalyptus no Brasil. Revista Árvore, Viçosa, v. 29, n. I, p. 129-137, 2005. 
HUANG, C.; HAN, L.; YANG, Z.; LIU, X. Ultimate analysis and heating value prediction of straw by near infrared spectroscopy. Waste Management, Oxford, v. 29, n. 6, p. 1793-1797, 2009.

KUMAR, R.; PANDEY, K. K.; CHANDRASHEKAR, N.; MOHAN, S. Effect of tree-age on calorific value and other fuel properties of Eucalyptus hybrid. Journal of Forestry Research, Tokyo, v. 21, n. 4, p. 514-516, 2010.

LIMA, E. A.; SILVA, H. D.; LAVORANTI, O. J. Caracterização dendroenergética de árvores de Eucalyptus benthamii. Pesquisa Florestal Brasileira, Colombo, v. 31, n. 65, p. 9-17, 2011.

LÓPEZ-RODRÍGUEZ, F; ATANET, C. P.; BLÁZQUEZ, F. C.; CELMA, A. R. Spatial assessment of the bioenergy potential of forest residues in the western province of Spain, Caceres. Biomass and Bioenergy, Oxford, v. 33, n. 10, p. I358-1366, 2009.

MINGOTI, S. A. Análise de dados através de métodos de estatística multivariada: uma abordagem aplicada. Belo Horizonte: UFMG, 2005. 297 p

MOGHTADERI, B.; SHENG, C.; WALL, T. F. An overview of the Australian biomass resources and utilization technologies. BioResources, Railegh, v. I, n. I, p. 93II5, 2006

MORI, F. A.; MENDES, L. M.; TRUGILHO, P. F.; CARDOSO, M. G. Utilização de eucaliptos e de madeiras nativas no armazenamento da aguardente de cana-de-açúcar. Ciência e Tecnologia de Alimentos, Campinas, v. 23, n. 3, p. 396400, 2003.

NEVES, T. A.; PROTÁSIO, T. P.; COUTO, A. M.; TRUGILHO, P. F; SILVA, V. O.; VIEIRA, C. M. M. Avaliação de clones de Eucalyptus em diferentes locais visando à produção de carvão vegetal. Pesquisa Florestal Brasileira, Colombo, v. 31 , n. 68 , p. $319-330,201$ I.

NEVES, T. A.; PROTÁSIO, T. P.; TRUGILHO, P. F.; VALLE, M. L. A.; SOUSA, L. C.; VIEIRA, C. M. Qualidade da madeira de clones de Eucalyptus em diferentes idades para a produção de bioenergia. Revista de Ciências Agrárias, Belém, v. 56 , n. 2, p. $139-148,2013$.

PAULA, L. E. R.; TRUGILHO, P. F.; NAPOLI, A.; BIANCHI, M. L. Characterization of residues from plant biomass for use in energy generation. Cerne, Lavras, v. I7, n. 2, p. 237-246, 2011 .

PROTÁSIO, T. P.; BUFALINO, L.; TONOLI, G. H. D.; COUTO, A. M.; TRUGILHO, P. F.; GUIMARÃES JÚNIOR, M. Relação entre o poder calorífico superior e os componentes elementares e minerais da biomassa vegetal. Pesquisa Florestal Brasileira, Colombo, v. 31, n. 66, p. 122-133, 2011 .
PROTÁSIO, T. P.; BUFALINO, L.; TONOLI, G. H. D.; GUIMARÃES JUNIOR, M.; TRUGILHO, P. F.; MENDES, L. M. Brazilian lignocellulosic wastes for bioenergy production: characterization and comparison with fossil fuels. BioResources, Railegh, v. 8, n. I, p. I|66-II85, $2013 a$.

PROTÁSIO, T. P.; COUTO, A. M.; REIS, A. A.; TRUGILHO, P. F. Seleção de clones de Eucalyptus para a produção de carvão vegetal e bioenergia por meio de técnicas univariadas e multivariadas. Scientia Forestalis, Piracicaba, v. 42, n. 97 , p. 15-28, 2013b.

PROTÁSIO, T. P.; TONOLI, G. H. D.; GUIMARÃES JÚNIOR, M.; BUFALINO, L.; COUTO, A. M.; TRUGILHO, P. F. Correlações canônicas entre as características químicas e energéticas de resíduos lignocelulósicos. Cerne, Lavras, v. I8, n. 3, p. 433-439, 2012a.

PROTÁSIO, T. P.; TRUGILHO, P. F.; NEVES, T. A.; VIEIRA, C. M. M. Análise de correlação canônica entre características da madeira e do carvão vegetal de Eucalyptus. Scientia Forestalis, Piracicaba, v. 40, n. 95, p. 317-326, 2012 b.

R DEVELOPMENT CORE TEAM. R: a language and environment for statistical computing. Vienna: $R$ Foundation for Statistical Computing, 2010. Disponível em: <http://www.R-project.org>. Acesso em: $10 \mathrm{dez}$. 2010.

REIS, A. A.; MELO, I. C. N. A.; PROTÁSIO, T. P.; TRUGILHO, P. F; CARNEIRO, A. C. Efeito de local e espaçamento na qualidade do carvão vegetal de um clone de Eucalyptus urophylla S. T. Blake. Floresta e Ambiente, Seropédica, v. 19, n. 4, p. 497-505, $2012 \mathrm{a}$.

REIS, A. A.; PROTÁSIO, T. P.; MELO, I. C. N. A.; TRUGILHO, P. F.; CARNEIRO, A. C. Composição da madeira e do carvão vegetal de Eucalyptus urophylla em diferentes locais de plantio. Pesquisa Florestal Brasileira, Colombo, v. 32, n. 7I, p. 277-290, 20I2b.

RODRIGUES, E. A. C.; ROSADO, S. C. S.; TRUGILHO, P. F.; SANTOS, A. M. Seleção de clones de Eucalyptus para as propriedades físicas da madeira avaliadas em árvores no campo. Cerne, Lavras, v. I4, n. 2, p. 147-I52, mar./abr. 2008.

SANTOS, R. C.; CARNEIRO, A. C. O.; CASTRO, A. F. M.; CASTRO, R. V. O.; BIANCHE, J. J.; SOUZA, M. M.; CARDOSO, M. T. Correlações entre os parâmetros de qualidade da madeira e do carvão vegetal de clones de eucalipto. Scientia Forestalis, Piracicaba, v. 39, n. 90, p. $22 \mathrm{I}-230,20 \mathrm{I}$.

SHARMA, R. K.; WOOTEN, J. B.; BALIGA, V. L.; LIN, X.; CHAN, W. G.; HAJALIGOL, M. R. Characterization of chars from pyrolysis of lignin. Fuel, London, v. 83, n. I I/I2, p. |469-I 482, 2004. 
SHENG, C.; AZEVEDO, J. L. T. Estimating the higher heating value of biomass fuels from basic analysis data. Biomass and Bioenergy, Oxford, v. 28, n. 5, p. 499-507, 2005.

TAN, Z.; LAGERLVIST, A. Phosphorous recovery from the biomass ash: a review. Renewable and Sustainable Energy Reviews, Amsterdam, v. I5, n. 8, p. 3588-3602, 201 I.

TELMO, C.; LOUSADA, J. Heating values of wood pellets from different species. Biomass and Bioenergy, Oxford, v. 35, n. 7, p. 2634-2639, $201 \mathrm{I}$.

THEODORO, V. C. A.; ALVARENGA, M. I. N.; GUIMARÃES, R. J.; SOUZA, C. A. S. Alterações químicas em solo submetido a diferentes formas de manejo do cafeeiro. Revista Brasileira de Ciência do Solo, Viçosa, v. 27, p. I039-1047, 2003.

TRUGILHO, P. F.; LIMA, J. T.; MENDES, L. M. Influência da idade nas características físico-químicas e anatômicas da madeira de Eucalyptus saligna. Cerne, Lavras, v. 2, n. I, p. 94 -I I I, 1996.

TRUGILHO, P. F.; LIMA, J. T.; MORI, F. A. Correlação canônica das características químicas e físicas da madeira de clones de Eucalyptus grandis e Eucalyptus urophylla. Cerne, Lavras, v. 9, n. I, p. 66-80, 2003.

TRUGILHO, P. F.; LIMA, J. T.; MORI, F. A.; LINO, A. L. Avaliação de clones de Eucalyptus para a produção de carvão vegetal. Cerne, Lavras, v. 7, n. 2, p. I04-II4, 200 I.
TRUGILHO, P. F.; VITAL, B. R.; REGAZZI, A. J.; GOMIDE, J. L. Aplicação da análise de correlação canônica na identificação de índices de qualidade da madeira de eucalipto para a produção de carvão vegetal. Revista Árvore, Viçosa, v. 21 , n. 2, p. 259-267, 1997.

VALE, A. T.; COSTA, A. F.; GONÇALEZ, J. C.; NOGUEIRA, M. Relações entre a densidade básica da madeira, o rendimento e a qualidade do carvão vegetal de espécies do cerrado. Revista Árvore, Viçosa, v. 25, n. 89, p. 8995, 2001 .

VARGAS-MORENO, J. M.; CALLEJÓN-FERRE, A. J.; PÉREZALONSO, J.; VELÁZQUEZ-MARTÍ, B. A review of the mathematical models for predicting the heating value of biomass materials. Renewable and Sustainable Energy Reviews, Amsterdam, v. I6, n. 5, p. 3065-3083, 2012.

VOIVONTAS, D.; ASSIMACOPOULOS, D.; KONKIOS, E. G. Assessment of biomass potential for power production: a GIS based method. Biomass and Bioenergy, Oxford, v. 20, n. 2, p. I0I-II2, $200 \mathrm{I}$.

YANG, H.; YAN, R.; CHEN, H.; ZHENG, C.; LEE, D. H.; LIANG, D. T. In-depth investigation of biomass pyrolysis based on three major components: hemicellulose, cellulose and lignin. Energy Fuels, Washington, v. 20, n. I, p. 388393, 2006. 